International Journal of Pure and Applied Mathematics

Volume 100 No. 3 2015, 379-383

ISSN: 1311-8080 (printed version); ISSN: 1314-3395 (on-line version)

url: http://www.ijpam.eu

doi: http://dx.doi.org/10.12732/ijpam.v100i3.4

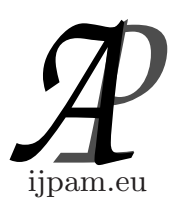

\title{
A CHARACTERIZATION OF
}

\section{PRIMITIVE ALGEBRAS VIA $B^{*}-$ SEMINORMS}

\author{
A.K. Gaur \\ Department of Mathematics \\ Duquesne University \\ Pittsburgh, PA 15282, USA
}

\begin{abstract}
Primitive algebras are characterized in terms of sub-seminorms. We prove a necessary and a sufficient condition for a $B^{*}-$ seminorm to be minimal. The results of this note are also valid for $M^{*}-$ seminorms introduced by Bhatt and others in [2].
\end{abstract}

AMS Subject Classification: 46H05, 47A10, 46H15

Key Words: $B^{*}-$ seminorms, minimal seminorms, primitive algebras

\section{Introduction}

Let $A$ be a complex $B^{*}$-algebra. For this algebra $A$, the norm is given by a $B^{*}-$ seminorm. A $B^{*}$ - seminorm on $A$ is a seminorm $p$ with the following conditions: For all $a, b \in A, p(a b) \leq p(a) \cdot p(b)$ and $p\left(a^{*} a\right)=(p(a))^{2}$. A seminorm $p$ on $A$ is an $M^{*}$ - seminorm if for all $a, b \in A, p(a b) \leq p(a) \cdot p(b)$ and $p\left(a^{*} a\right)=p(a)$. Clearly, every $B^{*}-$ seminorm on $A$ is an $M^{*}-$ seminorm on $A$ [2].

Let $N(A)$ be the set of all $B^{*}$ - seminorms on $A$. A partial ordering on $N(A)$ is defined as follows. For $p, q \in N(A), p \leq q$ if and only if $p(a) \leq q(a)$ for all $a \in A$. We say that a $B^{*}-\operatorname{seminorm} p$ on $A$ is a subseminorm if $p \in Q$, whenever $Q$ is a subset of $N(A)$ such that $p(a)=\max \{q(a): q \in Q\}$. The set of all sub-seminorms on $A$ is denoted by $N S(A)$.

Received: November 18, 2014

(c) 2015 Academic Publications, Ltd. url: www.acadpubl.eu 
Let $A$ be a complex Banach ${ }^{*}$-algebras where norm is a $B^{*}$ - seminorm on $A$. We call a $B^{*}$ - seminorm $p$ on $A$ is minimal if for a non-zero $p, p=q$, whenever $p$ is a nonzero $B^{*}$ - seminorm on $A$ dominated by $p$. Let $M N(A)$ denote the set of all minimal $B^{*}-$ seminorms on $A$. Then $M N(A) \subseteq N S(A)$.

Remark 1. We note that the norm on $A$ coincides with the largest $B^{*}-$ seminorm on $A$. To see this, let $g(a)=\sup \{p(a): p \in N(A), a \in A\}$. Then for all $a$ in $A,\|a\| \leq g(a)$. Also, $g(a) \leq\left\|a^{*} a\right\|^{1 / 2}=\|a\|$. Hence, $g(a)=\|a\|, \forall a \in$ $A$. This idea is also true in case of $M^{*}-$ seminorms, see [2].

We denote by $I_{d}(A)$ the set of all ideals of $A$. The set of primitive ideals and maximal ideals of $A$ that are written as $P R(A)$ and $M(A)$ respectively.

\section{Minimal Norms and Maximal Ideals of $A$}

In the following proposition, we present a characterization of minimal norms on $A$ in terms of maximal ideal space of $A$.

Proposition 1. A $B^{*}$ - seminorm $p$, is minimal if and only if $M_{p}$ is a maximal ideal.

Proof. For each $B^{*}-\operatorname{seminorm} p$ on $A$, let $M_{p}=\{a \in A: p(a)=0\}$. Then the quotient star algebra $A / M_{p}$ with the norm $\|\cdot\|_{p}$ is a $B^{*}$ - algebra, say $B_{p}$. Let $a \rightarrow \lambda_{a}$ be the canonical map from $A$ onto $A / M_{p}$. Then for all $\lambda_{a} \in B_{p}$, $\left\|\lambda_{a}\right\|_{p}=\inf \left\{\|a+x\|: x \in M_{p}\right\}$. We write $\bar{p}\left(\lambda_{a}\right)=p(a)$. Since, $\bar{p}$ is a $B^{*}-$ seminorm on $B_{p}$, we have by Remark $1, \bar{p}\left(\lambda_{a}\right) \leq\left\|\lambda_{a}\right\|_{p}$.

Further, by (1.8.1) in [5] $\left\|\lambda_{a}\right\|_{p} \leq p\left(\lambda_{a}\right)$. Thus $p(a)=\inf \{\|a+x\|: x \in$ $\left.M_{p}\right\}, a \in A$.

Suppose that $I$ is an ideal in $A$ and $A / I$ is the quotient $B^{*}$-algebra with the quotient norm $\|\cdot\|_{Q}$. Then a functional $p$ on $A$ can be defined by $p(a)=\|$ $\lambda_{a} \|_{Q}, a \in A$. This functional $p$ is a $B^{*}-\operatorname{seminorm} p$ on $A$. Also, $I=I_{d}(A)$. Hence the mapping $p \rightarrow I_{p}$ maps $N(A)$ onto $I_{d}(A)$. Furthermore, if $p$ and $q$ are in $\mathrm{N}(\mathrm{A})$ with $p \leq q$, we have $I_{q} \leq I_{p}$.

Next, we will show that if $p, q \in N(A)$ with $I_{q} \subseteq I_{p}$ then $p(a) \leq q(a), a \in A$. Since $p(a) \leq \inf \left\{\|a+x\|: x \in I_{q}\right\}$, we have $p(a) \leq q(a)$. Thus we have shown that the mapping $p \rightarrow I_{p}$ is one to one and $p$ is minimal if and only if $M_{p}$ is a maximal ideal. In fact, we have established that $p \rightarrow I_{p}$ maps $M N(A)$ one to one onto $M(A)$. 
Definition 1. a A nonzero $B^{*}-\operatorname{seminorm} p$ is external if $I_{p}$ is a prime ideal of $A$.

b With the null kernel topology, $P R(A)$ is called the structure space of $A$. The structure space is a locally compact $T_{o}$ space whose points are the $T_{1}$ elements of $M(A)$. Thus it is a $\pi$ - space if and only if $M(A)=P R(A)$.

Proposition 2. Let $p \in N S(A), p \neq 0$. The map $p \rightarrow I_{p}$ is a continuous one to one mapping from the set of all such $B^{*}$ - seminorms under the pointwise convergence topology, into the structure space of $A$.

Proof. Let $\widehat{A}$ be the set if equivalence classes of non-zero topologically irreducible star-representations of $A$. If $p \in N S(A)$, then there exists a $\pi \in \widehat{A}$ such that $p(a)=|\pi(a)|$. With this, $I_{p}$ is a primitive ideal because $\operatorname{ker} \pi=I_{p}$. Thus $N S(A) \backslash\{0\} \rightarrow P R(A)$ is a one to one map.

Let $F: N S(A) \backslash\{0\} \rightarrow P R(A)$ be the mapping defined by $p \rightarrow I_{p}$. Suppose that $S$ is a subset of $P R(A)$, which is closed in the null-kernel topology. Let $\left\{p_{\lambda}\right\}$ be a net in $F^{-1}(S)$ such that $p_{\lambda} \rightarrow p$. Since $I_{p_{\lambda}} \in S$, for all $\lambda$, we have $I_{p} \supseteq \bigcap\left\{I_{p_{\lambda}}\right\}$. Since $S$ is closed, it follows that $I_{p} \in S$. Thus $p \in F^{-1}(S)$ and $F^{-1}(S)$ is closed in the topology of pointwise convergence.

Definition 2. An algebra $A$ is said to be primitive if the mapping $p \rightarrow I_{p}$ defines an onto map.

The following theorem establishes a necessary and sufficient condition for $A$ to be Primitive.

Theorem 1. The algebra $A$ is primitive if and only if for each $\pi \in \widehat{A}$, the $B^{*}-$ seminorm $p_{\pi}$ is a sub nonzero seminorm on $A$.

Proof. For each $\pi \in \widehat{A}$, define the $B^{*}-\operatorname{seminorm} p_{\pi}(a)=|\pi(a)|, a \in A$. Suppose that $A$ is primitive, then $\operatorname{ker}(\pi)=I_{p_{\pi}}$. Hence, $p_{\pi}$ is a nonzero seminorm because $I_{p_{\pi}} \in P R(A)$.

To prove the converse, let $p_{\pi}$ be in $N S(A)$ and $I_{p} \in P R(A)$. Then there exists $\pi \in \widehat{A}$ with ker $=I_{p}$. This means that $I_{p_{\pi}}=I_{p}, p=p_{\pi}$, and $p \in$ $N S(A)$.

Corollary 1. If the structure space of $A$ is $T_{1}$, then $A$ is a primitive algebra. 
Proof. Let $I_{p}$ be a primitive ideal in $A$ for some $p$ in $N(A)$. Since $P R(A)$ is a $T_{1}$-space, we have $I_{p} \in M(A)$. Hence by Theorem $1, p$ is minimal and therefore sub and nonzero. Thus, $A$ is primitive.

Remark 2. If $A$ has a $T_{1}$ - structure space then the nonzero sub seminorms are minimal $B^{*}$ - seminorms. Further, if $A$ is primitive then the bijection map $F$ (which is continuous) is not a homeomorphism.

The following theorem gives a necessary and a sufficient condition for the map $p \rightarrow I_{p}$ to be a homeomorphism.

Theorem 2. The map $p \rightarrow I_{p}$ is a homeomorphism if and only if the structure space of $A$ is a $T_{2}-$ space.

Proof. Let the structure space, $P R(A)$ be a $T_{2}$ - space. The $P R(A)$ is also a $T_{1}$ - space and the map $F: N S(A) \backslash\{0\} \rightarrow P R(A)$ is one to one and onto. Denote the set of all closed subsets of $\operatorname{PR}(A)$ by $C l(A)$. The space $C l(A)$ is a compact $T_{2}-$ space. Define the map $\bar{F}: N(A) \rightarrow C l(A)$ by $\bar{F}(p)=\bar{I}_{p}$. Then by Theorem 2.2 in $[6] \bar{F}$ is a homeomorphism.

Next, we define the map $\phi: P R(A) \rightarrow C l(A)$ by $\phi\left(I_{p}\right)=\left\{I_{p}\right\}$. Then by [1], the map $\phi: P R(A) \rightarrow \phi(P R(A))$ is a homeomorphism. Our main objective is to show that the map $F$ is open. Suppose $G=\phi \circ F$. Then $G$ is well defined. Let $V$ be an open subset of $N S(A) \backslash\{0\}$. Then for an open set, $U$, in $N(A), V$ can be written as $V=U \cap(N S(A) \backslash\{0\})$. Therefore, $G(V)=\bar{F}(U) \cap \phi(P R(A))$ is open in $\phi(P R(A))$ because $\bar{F}(U)$ is open in $\overline{P R(A)}$. We also note that $\phi^{-1}(G(V))$ is open in $P R(A)$ since $\phi$ is a homeomorphism onto its range. In fact, $F(V)=\phi^{-1}(G(V))$ and $F(V)$ is open. Accordingly, $F$ is a homeomorphism.

Remark 3. In 2006, [2] the authors have used a similar seminorm, $M^{*}$ defined on a $*$ - subalgebra of a given $*$ - algebra. They have shown that an $M^{*}$-seminorm in unbounded if an $*$ - representation of $A$ exists. More on this to [3] [4]. The results of this paper hold for $M^{*}$-seminorms.

Remark 4. A $B^{*}$ - seminorm $p$ on $A$ is extremal if, whenever $q, r$ are $B^{*}$ seminorms on $A$ with $p(a)=\max \{r(a), q(a)\}, a \in A$ we have $p=q$ or $p=r$. Let $E N(A)$ denote the set of all extremal $B^{*}$ - seminorms on $A$. Then we have 
the following

$$
M N(A) \subseteq N S(A) \subseteq E N(A)
$$

It is easy to show that a nonzero $B^{*}$ - seminorm $p$ is extremal if and only if $I_{p}$ is a prime ideal. The mapping $p \rightarrow I_{p}$ maps $E N(A) \backslash\{0\}$ one to one onto $\operatorname{PR}(\mathrm{A})$.

\section{References}

[1] J.F. Aarnes, E.G. Effros, O.A. Nielsen, Locally compact spaces and two classes of $C^{*}$-algebras, Pacific J. Math, 34 (1970), 1-16, MR0271745.

[2] S.J. Bhatt, M. Fragoulopoutou, A. Inoue, Existence of spectral well behaved *- representations, J. of Math. Anal., 317 (2006), 475-495, MR2209574.

[3] S.J. Bhatt, A. Inoue, K.D. Kürsten, Well behaved unbounded operation *- representations and unbounded $C^{*}-$ seminorms, J. Math. Soc. Japan, 56 (2004), 417-445, MR2048467.

[4] S.J. Bhatt, A. Inoue, H. Ogi, Unbounded $C^{*}-$ seminorms and unbounded $C^{*}$ - spectral algebras, J. Operator Theory, 45 (2001), 53-80, MR1823062.

[5] J. Dixmier, Les $C^{*}-$ Algebras at Leurs Represantions, Gauthier-Villars, 1964.

[6] J.M.G. Fell, The structure of algebras of operator fields, Acta Math., 106 (1961), 233-280, MR0164248. 
